\section{Avaliação Dos Efeitos Da Fotobiomodulação Por Laser De Baixa Intensidade Em Pacientes Com Dor Lombar Crônica: Revisão Integrativa}

\author{
Evaluation Of The Effects Of Low Intensity Photobiomodulation On \\ Pain In Patients With Chronic Lumbar Pain: Integrative Review
}

\author{
Amanda Rocha Saraiva, Ivana De Alencar Calvacante, Diego Rodrigues Pessoa
}

\section{RESUMO:}

Objetivo: Introdução: A dor lombar crônica (DLC) é uma das causas de incapacidade osteomusculares sendo caracterizada como a principal afecção crônica existente em todo o mundo. Atualmente, diversos recursos de eletrotermofototerapêuticos vem sendo investigados com intuito de promover 0 alívio dos sintomas álgicos dos pacientes diagnósticos com DLC. Recentemente, a fotobiomodulação (FBM) por laser de baixa intensidade (LBI) e/ou luz intensa pulsada (LED) vem se demonstrando como um recurso amplamente eficaz para tratar dos sinais e sintomas da DLC, devido aos seus efeitos fotobiomoduladores. Objetivo: Avaliar os efeitos da FBM por LBI sobre a dor e incapacidade funcional em pacientes com DLC. Metodologia: $O$ presente estudo se caracteriza como revisão integrativa. A presente revisão foi realizada de acordo com as recomendações do Reporting Items for Systematic Reviews and Meta-Analyses (PRISMA). Foram considerados elegíveis os estudos publicados na língua inglesa. Os termos de pesquisa utilizados foram: "photobiomodulation therapy" or "low level laser" or "photherapy" or "photobiomodulation" and "low back pain" or "level back pain" or "back pain", os quais foram pesquisados nas bases de dados eletrônicas da PubMed/MedLine e PEDro. Resultados: Foram encontrados um total de 312 estudos com base nos descritores utilizados. Após a leitura dos títulos, resumos e estabelecimento dos critérios de elegibilidade, restaram apenas 7 estudos. Os autores citados nesta revisão integrativa evidenciaram que a FBM por LBI e/ou LED apresentaram efeitos satisfatórios, os quais podem favorecer satisfatoriamente a redução do quadro doloroso e melhorar a capacidade funcional dos pacientes diagnosticados com DLC. Conclusão: As partir dos dados obtidos por este estudo pode se concluir que a terapia a laser de baixa intensidade na intervenção fisioterapêutica empregada aos pacientes com lombalgia foi significativa na dor e na incapacidade funcional quando aplicada com uma dosimetria correta. Esse tratamento pode ser considerado uma opção terapêutica na reabilitação para dor lombar ou lombalgia.

PALAVRAS-CHAVE: Fotobiomodulação por laser de baixa intensidade; Dor lombar crônica; Incapacidade Funcional.

\section{ABSTRACT}

Objective: Introduction: Chronic low back pain (DLC) is one of the causes of musculoskeletal disability and is characterized as the main chronic condition existing worldwide. Currently, several electrothermal and phototherapeutic resources have been investigated in order to promote the relief of pain symptoms in patients diagnosed with DLC. Recently, photobiomodulation (FBM) by low-level laser (LBI) and / or intense pulsed light (LED) has been shown to be a widely effective resource for treating the signs and symptoms of DLC, due to its photobiomodulatory effects. Objective: To evaluate the effects of FBM by LBI on pain and functional disability in patients with DLC. Methology: The present study is characterized as an integrative review. This review was carried out in accordance with the recommendations of the Reporting Items for Systematic Reviews and Meta-Analyzes (PRISMA). Studies published in the English language were considered eligible. The search terms used were: "photobiomodulation therapy" or "low level laser" or "photherapy" or "photobiomodulation" and "low back pain" or "level back pain" or "back pain", which were searched in the databases electronic data from PubMed / MedLine and PEDro. Results: A total of 312 studies were found based on the keywords used. After reading the titles, abstracts and establishing the eligibility criteria, only 7 studies remained. The authors cited in this integrative review showed that FBM by LBI and / or LED had satisfactory effects, which can satisfactorily favor the reduction of the painful condition and improve the functional capacity of patients diagnosed with DLC. Conclusion: From the data obtained by this study, it can be concluded that low-level laser therapy in the physiotherapy intervention used in patients with low back pain was significant in pain and functional disability when applied with a correct dosimetry. This treatment can be considered a therapeutic option in rehabilitation for low back pain or low back pain.
Conflito de Interesse: Não há conflito de interesse 


\section{INTRODUÇÃO}

A dor lombar crônica (DLC) é uma das condições mais frequentes de dor crônica em todo o mundo, tornandose um grave problema de saúde pública¹. No Brasil, a lombalgia é uma das principais causas de incapacidade musculoesquelética, e um dos principais fatores que contribui para diminuição da incapacidade no trabalho². A DLC é a principal causa de incapacidade em todo o mundo, a qual é caracterizada por dor e desconforto localizado abaixo da margem costal e acima da linha glútea inferior, com ou sem dor referida, de forma prolongada ou de curta duração, variando com sua intensidade e duração, podendo apresentar uma recorrência dos casos clínicos entre $30 \%$ a $60 \%{ }^{3-5}$.

A lombalgia pode atingir $65 \%$ de indivíduos durante um ano, e $84 \%$ em algum momento da sua vida, com a prevalência proximamente de $11,9 \%$ da população mundial 6 . No Brasil, o problema crônico de coluna também representa uma das doenças crônicas mais frequentes na população, sendo a prevalência em adultos de 18 anos ou mais de 18,5\% (IC95\%: 17,8-19,1), segundo dados da Pesquisa Nacional de Saúde (PNS). O conhecimento da doença é por faixas etárias de idades, da gravidade do problema, do impacto na qualidade de vida, para promoção e prevenção dos problemas relacionado a dor na coluna na população ${ }^{7}$.

Os principais fatores etiopatogênicos da DLC são caraterizados por condições de origem como congênitas, degenerativas, inflamatórias, infecciosas, tumorais e mecânicos-posturais ${ }^{3}$. Alguns fatores podem contribuir para o surgimento da DLC, entre os quais, destacam-se: o estresse, alterações psicossociais, inatividade física, flexão lombar contínua, com ou sem rotação, em pé ou sentado ${ }^{8}$. A lombalgia está ligada ao processo de irritação das raízes nervosas da região lombar desencadeado de um processo degenerativo das articulações provocando um aumento da curvatura da coluna e fraqueza abdominal ${ }^{9}$. A dor lombar apresenta um conjunto de causa como, por exemplo fatores laborais, obesidade, síndromes depressivas, hábitos posturais entre outros. Através dessas alterações ocorre impacto anatomofuncional afetando a qualidade de vida e capacidade física ${ }^{10}$. Hábitos posturais como longos períodos em uma posição inadequada, utilização de saltos altos, nível de atividade física ou sedentários demonstra alterações na curvatura lombar ${ }^{11-14}$.

Atualmente na prática clínica, muitos tratamentos vêm sendo estudados com intuito de tratar os sinais e sintomas oriundos da DLC, entre os quais, destaca-se os tratamentos farmacológicos baseados no uso de fármacos antiinflamatório analgésico ${ }^{15}$, antidepressivos ${ }^{16}$, fitoterápicos ${ }^{17}$. Em relação aos tratamentos não farmacológicos, destacamse os tratamentos fisioterapêuticos os quais consiste no emprego da cinesioterapia ${ }^{18}$, auriculoterapia ${ }^{19}$, hidroterapia $^{20}$, crioterapia $^{21}$, estimulação elétrica nervosa transcutânea ${ }^{22}$.

Recentemente, a fotobiomodulação (FBM) por laser de baixa intensidade (LBI) e/ou luz diodo emissor (LED) vem ganhando grande destaque para o tratamento de distúrbios osteomusculares, devido aos seus efeitos fotobiomoduladores 
(anti-edematoso, anti-cicatricial e anti-inflamatório). O termo FBM corresponde ao emprego luz monocromáticas (laser e LEDs) para realizar a modulação de processos bioquímicos relacionados ao metabolismo celular ${ }^{23,24,25,26}$.

A FBM pode ser empregada dentro da janela terapêutica, podendo ser utilizada no espectro eletromagnético do vermelho (623,8 a $700 \mathrm{~nm}$ ) e infravermelho (700 a $1064 \mathrm{~nm})$. A FBM quando empregada com a dosimetria adequada (potência, energia, densidade de energia, densidade de potência e tempo) favorecem os estímulos fotobiomoduladores, os quais podem por penetrar os tecidos, exercendo modificações na síntese, liberação e modulação do processo inflamatório e no controle da analgesia $23,24,25,26$.

Os efeitos da estimulação da FBM no tecido são alterações na permeabilidade da membrana, vasodilatação, redução do edema celular e bloqueio das fibras nervosas em resposta à liberação de opióides endógenos (endorfinas e encefalinas). Bem como a diminuição da liberação de histamina, acetilcolina e síntese bradicinesia. Dessa forma essa alteração tem como resposta à redução da dor, a produção de cicloxigenase 2 (COX-2), prostaglandinas e secreções de histamina, citocinas e cininas, como fator de necrose tumoral alfa (TNF-a), fator de crescimento transformador-beta (TGF- $\beta$ ) e interleucinas IL-1 $\beta$ e IL-6 $6^{23}$.

O presente trabalho está inserido no contexto de estudar as percepções da ação da FBM como uma recurso terapêutico complementar no tratamento da DLC, uma vez que acredita-se que o método possa favorecer a redução da dor e melhorar a qualidade de vida, devido aos seus efeitos analgésicos, anti-inflamatórios e reparador tecidual com modulação de atividade celular. Diante do apresentado, o presente estudo tem como objetivo realizar revisão integrativa acerca dos efeitos da FBM por LBI e/ou LED em pacientes diagnosticados com dor lombar crônica (DLC)

\section{MÉTODO}

O presente estudo se caracteriza como uma revisão integrativa de literatura, a qual investigou os efeitos da FBM por LBI e/ou LED sobre a dor e capacidade funcional de pacientes diagnosticados com DLC. A presente revisão foi pesquisada artigos nas bases de dados eletrônicas da MedLine/PubMed, e PEDro entre os meses de agosto de 2020 e outubro de 2020. Foram considerados apenas artigos elegíveis na língua inglesa. Os descritores de pesquisa utilizados foram: "Low back pain" OR "back pain" AND "photobiomodulation therapy" OR "low level laser therapy" OR "photobiomodulation" OR "phototherapy" AND "clinical trial" OR "controlled randomized trial" OR "trial".

Os artigos encontrados foram submetidos a leitura dos resumos e confrontados de acordo com os critérios de inclusão e exclusão dessa revisão. Como critérios de inclusão, foram considerados os seguintes preceitos: (1) artigos publicados no idioma inglês sem restrição de período cronológico (2) estudos controlados e randomizados (3) publicações que enfatizavam os efeitos da FBM sobre a dor e capacidade funcional na DLC. Já como critério de exclusão 
foram adotados os seguintes preceitos: (1) Estudos de caso, revisão de literatura, bibliográficas, revisão sistemática com metanálise, teses e dissertações e (2) artigos por repetição e (3) artigos não disponíveis para download nas bases de dados.

As autoras (A.R.S) e (I.A.C) selecionaram os estudos para inclusão baseados na leitura e análise crítica dos títulos e resumos. Os artigos foram classificados em pesquisas adequadas (quando os estudos estavam adequados aos critérios de elegibilidade) e inadequadas (os que não se enquadram nos objetivos propostos). Os dados dos estudos foram analisados e tabulados em uma tabela pré-estabelecida os quais compreendem os seguintes itens: autor/ano, revista publicada, objetivo, amostra, protocolo metodológico, instrumentos e resultados.

\section{RESULTADOS}

Figura 1: Fluxograma de seleção e identificação dos estudos.
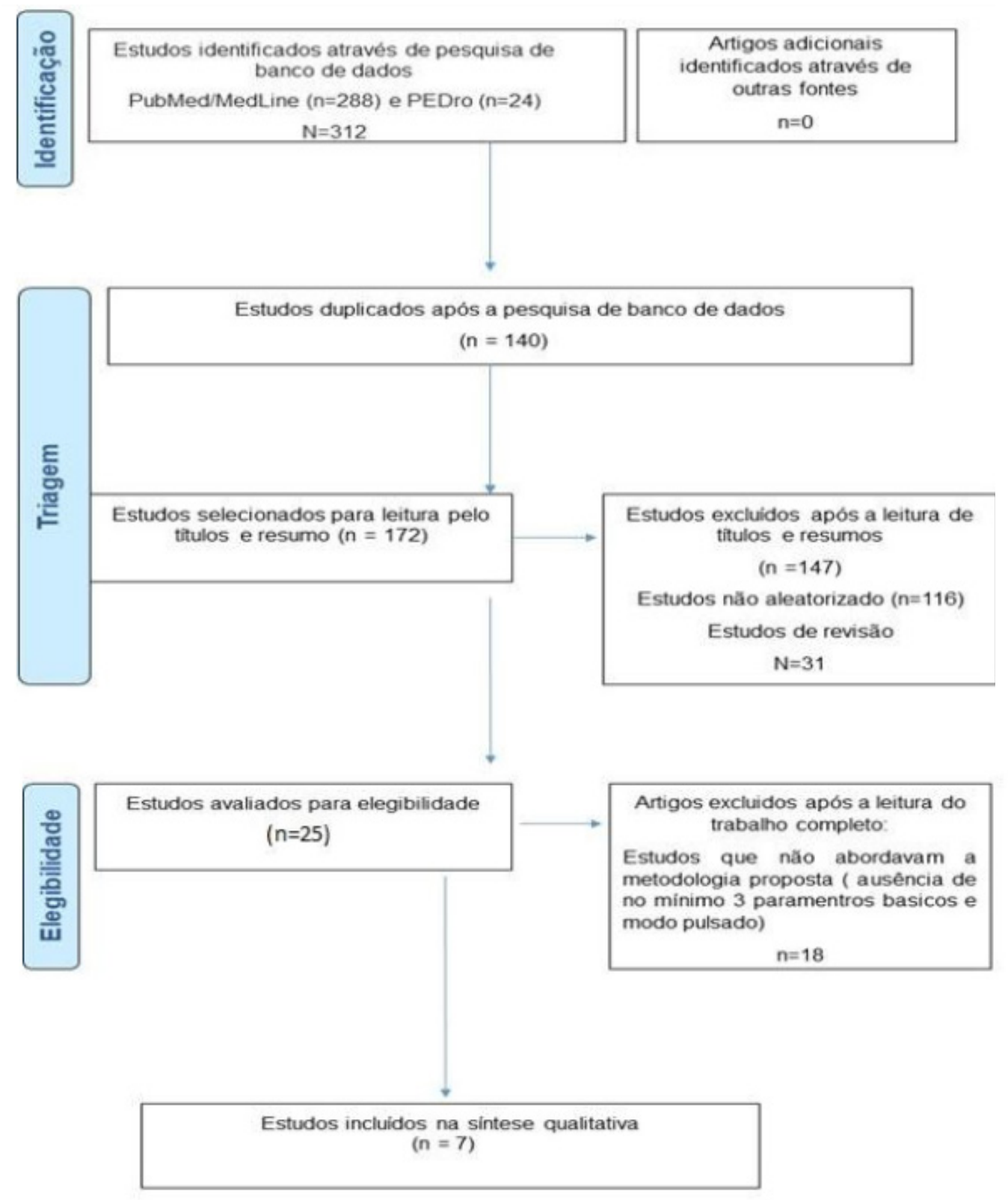

publicada, objetivo, amostra, protocolo metodológico e resultados.

Quadro 1: Estudos incluídos na revisão, organizados em ordem cronológica de publicação: autores/ano, objetivo, amostra, protocolo metodológico, instrumentos e resultados.

\begin{tabular}{|c|c|c|c|c|c|c|}
\hline $\begin{array}{l}\text { Autorl } \\
\text { ano }\end{array}$ & Revista & Objetivo & Amostra & Protocolo metodológico & Instrumento & Resultados \\
\hline $\begin{array}{l}\text { Basford; } \\
\text { (1999) }\end{array}$ & $\begin{array}{l}\text { Archives of } \\
\text { Physical } \\
\text { Medicine and } \\
\text { Rehabilitation }\end{array}$ & $\begin{array}{l}\text { Avaliar a eficácia do } \\
\text { FBM por LBI no } \\
\text { tratamento da DLC }\end{array}$ & $\begin{array}{c}56 \\
\text { participantes }\end{array}$ & $\begin{array}{l}\text { Grupo controle: os pacientes } \\
\text { foram tratados com FBM de } \\
\text { forma que o aparelho } \\
\text { permaneceu inativo. } \\
\text { Grupo intervenção: os } \\
\text { pacientes foram tratados com } \\
\text { FBM na respectiva } \\
\text { dosimetria } \\
\text { (P: } 542 \text { mW; } \lambda: 1064 \mathrm{~nm} \text {; T: } \\
90 \text { s), durante } 4 \text { semanas. }\end{array}$ & $\begin{array}{c}\text { Questionário de } \\
\text { deficiência de } \\
\text { Oswestry } \\
\text { Escalas visuais } \\
\text { analógicas (VAS). }\end{array}$ & $\begin{array}{c}\text { Os resultados dos } \\
\text { estudos apontaram que o } \\
\text { tratamento com FBM } \\
\text { grupo intervenção obteve } \\
\text { uma redução moderada } \\
\text { da dor comparado com o } \\
\text { grupo controle, no } \\
\text { entanto foram limitados e } \\
\text { diminuindo com o tempo. }\end{array}$ \\
\hline $\begin{array}{c}\text { Glazov; et } \\
\text { al. } \\
(2009)\end{array}$ & $\begin{array}{l}\text { Acupuncture in } \\
\text { Medicine }\end{array}$ & $\begin{array}{l}\text { Comparar os efeitos do } \\
\text { laser acupuntural (ativo } \\
\text { x placebo) na redução } \\
\text { da dor e incapacidade } \\
\text { em adultos com dor } \\
\text { lombar crônica } \\
\text { (inespecifica) }\end{array}$ & $\begin{array}{c}90 \\
\text { participantes }\end{array}$ & $\begin{array}{l}\text { Grupo Placebo: os pacientes } \\
\text { foram tratados com FBM de } \\
\text { forma em que o aparelho } \\
\text { permaneceu desligado. } \\
\text { Grupo Intervenção: os } \\
\text { pacientes foram tratados com } \\
\text { FBM na respectiva } \\
\text { dosimetria (P: } 10 \mathrm{~mW} ; \lambda: 830 \\
\mathrm{~nm} ; \mathrm{P}: 0.2 \mathrm{~J} \text {; DP:0.05 W/cm } 2 \\
\text { e T: } 20 \mathrm{~s} \text {. Os pacientes foram } \\
\text { submetidos a } 10 \text { sessões de } \\
\text { tratamento. }\end{array}$ & $\begin{array}{c}\text { Escala visual } \\
\text { analógica (EVA); } \\
\text { Îndice de deficiência } \\
\text { de Oswestry. }\end{array}$ & $\begin{array}{l}\text { Os resultados do estudo } \\
\text { apontaram que houve } \\
\text { uma melhora da dor e } \\
\text { incapacidade funcional } \\
\text { nos grupos tratados, } \\
\text { entretanto, o tratamento } \\
\text { com a FBM (ativo) não } \\
\text { foi superior ao placebo, } \\
\text { nos parâmetros } \\
\text { utilizados. }\end{array}$ \\
\hline $\begin{array}{l}\text { Ay, } \\
\text { Doğan, } \\
\text { Evcik, } \\
(2010)\end{array}$ & $\begin{array}{c}\text { Clinical } \\
\text { Rheumatology }\end{array}$ & $\begin{array}{l}\text { Comparar a eficácia da } \\
\text { FBM na dor e } \\
\text { capacidade funcional } \\
\text { em pacientes } \\
\text { com dor DLC } \\
\text { (aguda/crônica) }\end{array}$ & $\begin{array}{c}80 \\
\text { participantes }\end{array}$ & $\begin{array}{l}\text { Grupo } 1(\mathrm{LDH} \text { aguda, } n=20) \\
\text { recebeu embalagem quente } \\
+ \text { laser terapia. } \\
\text { Grupo } 2 \text { (LDH aguda, } n=20 \text { ) } \\
\text { recebeu embalagem quente } \\
\text { + placebo terapia a laser. } \\
\text { Grupo } 3 \text { (LDH crônica, } n= \\
\text { 20) recebeu embalagem } \\
\text { quente + laser terapia. } \\
\text { Grupo } 4 \text { (LDH crônica, } n= \\
20) \text { recebeu embalagem } \\
\text { quente + terapia a laser com } \\
\text { placebo; os pacientes foram } \\
\text { tratados por FBM nas } \\
\text { respectivas doses: } \lambda: 850 \mathrm{~nm} \text {; } \\
100 \mathrm{~mW} ; 40 \mathrm{~J} / \mathrm{cm}^{2} \text { ). }\end{array}$ & $\begin{array}{c}\text { Escala visual } \\
\text { analógica (EVA); } \\
\text { Disability Roland } \\
\text { Disability } \\
\text { Questionnaire (RDQ); } \\
\text { Questionário de } \\
\text { Deficiência de } \\
\text { Oswestry (ODQ). }\end{array}$ & $\begin{array}{c}\text { Os resultados dos } \\
\text { estudos mostram que } \\
\text { embora todos os grupos } \\
\text { tenham mostrado } \\
\text { melhorias nos } \\
\text { parâmetros de avaliação, } \\
\text { não conseguiram mostrar } \\
\text { a superioridade à FBM } \\
\text { em relação ao laser } \\
\text { placebo na dor, } \\
\text { gravidade e capacidade } \\
\text { funcional em pacientes } \\
\text { com DLC } \\
\text { (agudo/crônica). }\end{array}$ \\
\hline $\begin{array}{c}\text { Hsieh; Lee } \\
\text { (2013) }\end{array}$ & $\begin{array}{l}\text { Lasers in Medical } \\
\text { Science }\end{array}$ & $\begin{array}{l}\text { Investigar os efeitos da } \\
\text { terapia a laser por } 890 \\
\text { nm a curto prazo em } \\
\text { pacientes com DLC. }\end{array}$ & $\begin{array}{c}60 \\
\text { participantes }\end{array}$ & $\begin{array}{l}\text { Grupo placebo: os pacientes } \\
\text { foram tratados com FBM e } \\
\text { compressas quentes, mas o } \\
\text { dispositivo permaneceu } \\
\text { desligado. } \\
\text { Grupo intervenção: os } \\
\text { pacientes foram tratados com } \\
\text { embalagem quente e FBM na } \\
\text { respectiva dosimetria (13 } \\
\mathrm{mW} ; \lambda: 890 \mathrm{~nm} ; 6,24 \mathrm{~W} / \mathrm{cm}^{2} \text {, } \\
\left.83,2 \mathrm{~J} / \mathrm{cm}^{2}\right) \text { os pacientes } \\
\text { foram tratados. }\end{array}$ & $\begin{array}{c}\text { Escalas visuais } \\
\text { analógicas (EVA); } \\
\text { Classificação } \\
\text { Internacional de } \\
\text { Funcionalidade (CIF); } \\
\text { Escala de } \\
\text { Independência } \\
\text { Funcional - MIF } \\
\text { Questionário de } \\
\text { Deficiência de } \\
\text { Oswestry (ODQ). }\end{array}$ & $\begin{array}{l}\text { Os resultados do estudo } \\
\text { apontam que FBM } \\
\text { associado com } \\
\text { compressas quentes } \\
\text { reduziu a dor crônica na } \\
\text { parte inferior da lombar, } \\
\text { em comparação com } \\
\text { compressas quentes } \\
\text { combinada com terapia a } \\
\text { luz placebo. } \\
\text { Portanto, a redução da } \\
\text { dor foi associada às } \\
\text { reducões de gravidade }\end{array}$ \\
\hline
\end{tabular}




\begin{tabular}{|c|c|c|c|c|c|c|}
\hline & & & & $\begin{array}{c}\text { semanalmente por } 2 \\
\text { semanas com duração de } 40 \\
\text { min. }\end{array}$ & & $\begin{array}{c}\text { da deficiência e crenças } \\
\text { de medo de evitar a } \\
\text { atividade física. }\end{array}$ \\
\hline $\begin{array}{l}\text { Vallone; et } \\
\text { al. (2014) }\end{array}$ & $\begin{array}{c}\text { Photomedicine } \\
\text { and Laser } \\
\text { Surgery }\end{array}$ & $\begin{array}{l}\text { Avaliar a eficácia da } \\
\text { redução da dor de } \\
\text { tratamento com FBM } \\
\text { diodo Ga-AL-As } \\
(980 \mathrm{~nm}) \text { em } \\
\text { combinação com } \\
\text { terapia por exercicios, } \\
\text { em pacientes com } \\
\text { DLC. }\end{array}$ & $\begin{array}{c}100 \\
\text { participantes }\end{array}$ & $\begin{array}{l}\text { Grupo placebo: os pacientes } \\
\text { foram tratados com } \\
\text { exercícios diários e FBM de } \\
\text { forma que o aparelho } \\
\text { permaneça desligado. } \\
\text { Grupo intervenção: os } \\
\text { pacientes foram tratados com } \\
\text { exercícios diários e com FBM } \\
\text { na respectiva dosimetria: } \lambda \\
980 \mathrm{~nm} ;: 20 \mathrm{~mW} ; 37,5 \mathrm{~J} / \mathrm{cm}^{2} \text {; } \\
1 \mathrm{~min}) .\end{array}$ & $\begin{array}{c}\text { Escala visual } \\
\text { analógica (EVA). }\end{array}$ & $\begin{array}{l}\text { Os resultados do estudo } \\
\text { apontam que a FBM } \\
\text { diodo de } 980 \mathrm{~nm} \\
\text { combinada com o } \\
\text { programa de exercícios } \\
\text { se mostrou mais eficaz } \\
\text { na redução da dor } \\
\text { comparado ao grupo } \\
\text { placebo só exercícios, } \\
\text { podendo ser promissor } \\
\text { como uma ação } \\
\text { terapêutica na } \\
\text { reabilitação da DLC. }\end{array}$ \\
\hline $\begin{array}{l}\text { Doğan, } \\
\text { Ay, Evcik, } \\
(2017)\end{array}$ & $\begin{array}{l}\text { Journal of Back } \\
\text { and } \\
\text { Musculoskeletal } \\
\text { Rehabilitation }\end{array}$ & $\begin{array}{l}\text { Comparar a eficácia de } \\
\text { dois regimes diferentes } \\
\text { de FBM sobre dor, } \\
\text { amplitude de } \\
\text { movimento (ADM) } \\
\text { lombar e capacidade } \\
\text { funcional em pacientes } \\
\text { com DLC. }\end{array}$ & $\begin{array}{c}49 \\
\text { participantes }\end{array}$ & 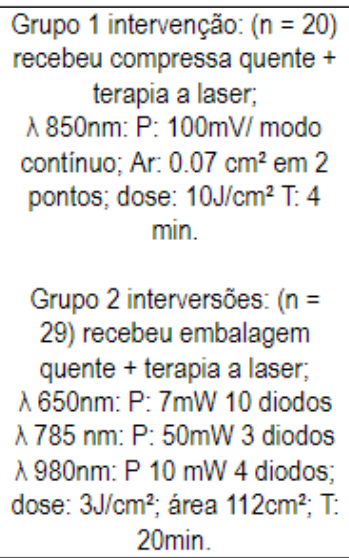 & $\begin{array}{c}\text { Escala visual } \\
\text { analógica (EVA); } \\
\text { Índice de deficiência } \\
\text { de Oswestry. }\end{array}$ & $\begin{array}{l}\text { Os resultados do estudo } \\
\text { apontaram que o uso de } \\
\text { diferentes aplicações da } \\
\text { FBM pode proporcionar } \\
\text { melhorias na ADM e na } \\
\text { capacidade funcional dos } \\
\text { pacientes com DLC, no } \\
\text { entanto não há } \\
\text { superioridade na } \\
\text { severidade da dor entre } \\
\text { estes dois tipos } \\
\text { especíicos de } \\
\text { modalidades da FBM. }\end{array}$ \\
\hline
\end{tabular}

\section{DISCUSSÃO}

Este estudo se propôs em realizar uma revisão integrativa baseada na leitura de artigos científicos internacionais acerca dos efeitos da FBM por LBI e/ou LED sobre a dor e incapacidade funcional em indivíduos com DLC. Segundo a Associação Internacional para Estudos da Dor (IASP), a dor pode ser definida como "uma experiência sensitiva e emocional desagradável associada a uma lesão tecidual real ou potencial, ou descrita nos termos de tal lesão"27. A DLC é uma condição altamente prevalente em todo o mundo e a principal causa de incapacidade em seus portadores, devido ainda os tratamentos não estarem bem estabelecidos pela literatura, optou-se em realizar o estudo envolvendo os efeitos da FBM por $\mathrm{LBI}^{28}$.

O presente estudo selecionou as principais evidencias científicas que estudaram os efeitos da FBM por LBI e/ou LED, onde foi comprovado que o método quando aplicado de forma isolada ou associados à outros recursos terapêuticos farmacológicos ou convencionais podem apresentar resultados satisfatórios sobre a dor. Diante disso, Basford et al. $(1999)^{29}$ investigaram os efeitos do FBM em indivíduos diagnosticado com DLC, em que os pacientes foram tratados com a seguinte dosimetria (P: $542 \mathrm{~mW} ; \lambda: 1064 \mathrm{~nm} ; \mathrm{T}: 90 \mathrm{~s}$ ). Ao fim do estudo os autores concluíram que a FBM quando empregados aos parâmetros citados, possibilitou a melhora do quadro doloroso no período de 4 semanas comparado com o grupo controle, tais resultados podem ser corroborados com estudo proposto por Kholoosy et al. (2020) ${ }^{30}$ os 
quais compararam os benefícios da radiação eletromagnéticas do infravermelho próximo ( $\lambda 808 \mathrm{~nm}$; P: $160 \mathrm{~mW}$; modo: contínuo; T:30 s; dose: 4,5 J/cm²) aplicados sobre a musculatura para-vertebral, assim como, nos pontos acupuntura com dose (1,5 j/cm²; T: 10seg;) e, foi comprovado que a irradiação no espectro eletromagnético favoreceu resultados satisfatórios sobre a dor quando comparado

Os resultados encontrados por Basford et al (1999) ${ }^{29}$ e Kholoosy et al (2020) 30 podem ser confirmados por Pessoa et al. $(2018)^{23}$ que confirmaram em seu estudo que a FBM tem a capacidade de reduzir a dor devido aos efeitos fotobiomoduladores que tendem a promover o aumento da circulação periférica, promovendo a quebra no ciclo dorespasmo-dor, redução do edema e por fim aumento no limiar da dor, uma vez que este está relacionado com a diminuição da velocidade de condução nervosa, devido a liberação dos opioides endógenos neurotransmissores essenciais para a redução da dor. Já Cotler et al. (2015) $)^{31}$ reafirmam que dosimetrias adequadas (comprimento de onda, potência, energia, densidade de energia/potência e tempo) podem influenciar satisfatoriamente sobre a dor.

Vallone et al. (2014) $)^{32}$ avaliaram a eficácia da FBM por LBI $(980 \mathrm{~nm})$ na redução da dor. Foi observado que quando administrada a dosimetria ( $\lambda 980 \mathrm{~nm}: 20 \mathrm{~mW} ; 37,5 \mathrm{~J} / \mathrm{cm}^{2} ; 1 \mathrm{~min}$ ) houve redução da dor nos grupos tratados com laser + exercícios comparado ao grupo placebo (apenas exercícios), o que leva a concluir que associação da FBM aos exercícios terapêuticos pode favorecer satisfatoriamente a redução do quadro álgico dos pacientes. Esses resultados corroboram com os estudos propostos por Vanin et al. $(2018)^{33}$ e Leal Junior et al. $(2019)^{34}$, os quais comprovaram que a FBM associada aos exercícios terapêuticos pode potencializar os efeitos satisfatórios reduzindo a dor e melhorando o nível de incapacidade funcional.

A DLC é um dos problemas de saúde pública mais comuns que proporciona elevados custos para o sistema único de saúde (SUS), uma vez que, dependendo do estágio pode se tornar uma importante causa de absenteísmo ao trabalho em todo o mundo, impactando negativamente sobre a saúde funcional dos seus portadores. Glazov et al. $(2009)^{35}$ e Huang, et al. (2015) ${ }^{36}$ investigaram os efeitos do tratamento com a FBM com laser acupuntural no espectro eletromagnético do infravermelho $(890 \mathrm{~nm})$ padronizado com a respectiva dosimetria (P: $10 \mathrm{~mW}$; $\lambda: 830 \mathrm{~nm}$; $P: 0.2 \mathrm{~J}$; DP:0.05 W/cm2 e T: $20 \mathrm{~s}$ ) e foi observado que ao fim do estudo que a FBM quando comparada a grupos placebos favorece melhora significativa sobre a incapacidade funcional.

Os achados de Glazov et al. (2009) $)^{35}$ e Huang, et al. $(2015)^{36}$ podem ser justificados pelos achados encontrados por Leal Junior et al. (2011) $)^{34}$ que comprovou que os comprimentos de onda dentro do espectro do infravermelhos tendem a ser mais eficazes quando comparados com o espectro do vermelho, uma vez que é comprovado que a FBM (infravermelha) favorece um aumento considerável da microcirculação periférica, o que propicia a redução satisfatória do espasmo muscular auxiliando na redução da dor e melhorando a capacidade funcional.

Ay, Doğan, Evcik, $(2010)^{37}$ compararam os efeitos da FBM por LBI ( $\lambda: 850 \mathrm{~nm}$; P: 100mv- contínuo; área 
pontual $0,07 \mathrm{~cm}^{2}$; energia $40 \mathrm{~J} / \mathrm{cm}^{2}$ ) em pacientes com DLC. Os resultados deste estudo não demonstraram resultados satisfatórios da FBM quando comparado ao placebo, não corroborando com os estudos de Glazov et al. (2009) ${ }^{35} \mathrm{e}$ Huang, et al. (2015) ${ }^{36}$. Acredita-se que tais resultados podem ter se apresentado não satisfatórios devido alguma falha na adequação do procedimento metodológico. Entretanto, Ay, Doğan, Evcik (2017) ${ }^{38}$ demonstrou em seu estudo que 0 uso dos diferentes tipos de aplicação do laser pode proporcionar ganhos de amplitude de movimentos e melhoria da incapacidade funcional dos pacientes.

As proposições enfatizadas por Hsieh et al. $(2013)^{39}$ podem defender os achados propostos por Ay, Doğan, Evcik, (2010) $)^{37}$, Huang, et al. (2015) ${ }^{36}$ e Ay, Doğan, Evcik $(2017)^{38}$ sobre os resultados obtidos na incapacidade funcional, pois acredita-se que os fatores psicológicos do paciente podem estar envolvidos na melhora do índice de incapacidade, uma vez que os sinais interpretativos da dor e a entrada nociceptiva para o córtex cerebral são complexos e à experiência subjetiva da dor pode fazer a diferença, sendo que o emprego da FBM por LBI e/ou LED podem favorecer na redução da sensibilidade cortical, especificamente, relacionadas ao processamento da dor, quais podem favorecer na redução da sensibilidade álgica, tornando o paciente mais ativo para realizar suas atividades ocupacionais prejudicadas.

Os resultados obtidos por este estudo são baseados na leitura dos artigos publicados por Basford et al (1999) ${ }^{29}$, Glazov et al. (2009) $)^{35}$, Ay, Doğan, Evcik (2010) $)^{37}$, Hsieh et al. (2013) $)^{39}$, Vallone et al. (2014) $)^{32}$, Ay, Doğan, Evcik (2017) ${ }^{38}$ e Kholoosy et al $(2020)^{30}$ tanto sobre a dor quanto a incapacidade funcional só foram satisfatórios devido a FBM também atuar sobre a liberação de óxido nítrico, com subsequentes alterações bioquímicas e fisiológicas sub-celulares e celulares, entretanto é de suma importância considerar que qualquer tratamento baseado na FBM seja por LBI e/ou LED para que aconteça os efeitos clínicos é necessário a padronização dos parâmetros dosimétricos, tais como: o comprimento de onda, a energia, a potência, a densidade de energia, o método de aplicação (modo de contato ou modo sem contato), o número total de sessões de tratamento, a frequência do tratamento e a duração de cada sessão de tratamento, visando os seus resultados satisfatórios.

O FBM por LBI e/ou LED associado com outras modalidades terapêuticas como o uso de AINEs, exercícios e compressas quentes do qual foram citados acima demonstraram que seus efeitos são positivos quando estão associados a terapia a laser de baixa intensidade, ou seja, as modalidades terapêuticas aplicadas sozinhas não se sobressaem a terapia.

\section{CONSIDERAÇÕES FINAIS}

A partir dos dados obtidos por este estudo de revisão integrativa permite concluir que a FBM por LBI e/ou LED's pode favorecer resultados satisfatórios sobre o controle da dor em pacientes diagnosticados com DLC, quando 
empregada com os parâmetros dosimétricos adequados (potência, comprimento de onda, energia e tempo).

\section{REFERÊNCIAS}

1. TOMAZONI, Shaiane Silva et al. Efeitos da terapia de fotobiomodulação em pacientes com dor lombar crônica inespecífica: protocolo para um estudo randomizado controlado com placebo. BMJ aberto, v. 7, n. 10, 2017

2. ALNAAMI, Ibrahim et al. Prevalence and factors associated with low back pain among health care workers in southwestern Saudi Arabia. BMC musculoskeletal disorders, v. 20, n. 1, p. 56, 2019.

3. PIRES, Renata Alice Miateli; DUMAS, Flavia Ventura Ladeira. Lombalgia: revisão de conceitos e métodos de tratamentos. Universitas: ciências da saùde, v. 6, n. 2, p. 159-168, 2008.

4. HELFENSTEIN Junior, M., Goldenfum, M. A., \& Siena, C. (2010). Lombalgia ocupacional. Revista Da Associação Médica Brasileira, 56(5), 583-589.

5. Will JS, Bury DC, Miller JA. Mechanical Low Back Pain. Am Fam Physician. 2018 Oct 1;98(7):421-428. PMID: 30252425.

6. NASCIMENTO, Paulo Roberto Carvalho do; COSTA, Leonardo Oliveira Pena. Prevalência da dor lombar no Brasil: uma revisão sistemática. Cadernos de saúde pública, v. 31, p. 1141-1156, 2015.

7. RAMPAZO, Érika Patrícia et al. Photobiomodulation therapy and transcutaneous electrical nerve stimulation on chronic neck pain patients: Study protocol clinical trial (SPIRIT Compliant). Medicine, v. 99, n. 8, 2020.

8. URITS, I., Burshtein, A., Sharma, M., Testa, L., Gold, P. A., Orhurhu, V., ... Kaye, A. D. (2019). Low Back Pain, a Comprehensive Review: Pathophysiology, Diagnosis, and Treatment. Current Pain and Headache Reports, 23(3).

9. ALMEIDA, Darlan Castro; KRAYCHETE, Durval Campos. Dor lombar-uma abordagem diagnóstica. Revista Dor, v. 18, n. 2, p. 173-177, 2017 https://doi.org/10.5935/1806-0013.20170034

10. VALADARES, Jessyka Viana et al. Prevalência da lombalgia e sua repercussão anatomofuncional em adultos e idosos: Revisão sistemática. AMAZÔNIA: SCIENCE \& HEALTH, v. 8, n. 3, p. 106-117, 2020.

11. DA SILVA, Michael Douglas SOMERLATE; CAMEY, Leonardo Ura; CUNHA, Rodrigo Gontijo. Dor e incapacidade na região lombar referente a lombalgia em alunos dos últimos períodos do curso de fisioterapia em uma instituição de ensino superior. Nbc-Periódico Científico do Núcleo de Biociências, v. 10, n. 19, 2020

12. ALLEGRI, Massimo et al. Mechanisms of low back pain: a guide for diagnosis and therapy. F1000Research, v. 5, 2016.

13. POSSEBOM, Michele; BERNARDI, Diogo Luiz; ALVES, Izabel Almeida. Lombalgia: o papel do farmacêutico 
na identificação, acompanhamento, manejo e prevenção. Revista interdisciplinar em ciências da saúde e biológicas-ricsb, v. 3, n. 1, p. 86-100, 2019. DOI: 10.31512/ricsb. v3i1.2932

14. RIBEIRO, Renilde Carlos; MARTINS, Patrícia Cândida de Matos Lima; PEREZ, Fabiana da Silva Bianchi. Cinesioterapia no tratamento da dor lombar crônica: revisão de literatura. Saúde \& ciência em ação, v. 5, n. 1, p. 82-91, 2019.

15. Casiano VE, Dydyk AM, Varacallo M. Back Pain. [Updated 2020 Jul 10]. In: StatPearls [Internet]. Treasure Island (FL): StatPearls Publishing; 2020 Jan-. Available from: https://www.ncbi.nlm.nih.gov/books/NBK538173/COTLER, Howard B. et al. The use of low level laser therapy (LLLT) for musculoskeletal pain. MOJ orthopedics \& rheumatology, v. 2, n. 5, 2015.

16. URQUHART, Donna M. et al. Antidepressants for non[specific low back pain. Cochrane Database of Systematic Reviews, n. 1, 2008.

17. GAGNIER, Joel J. et al. SPINE An International Journal for the study of the spine Publish Ahead of Print. 2015. DOI : 10.1097/BRS.0000000000001854

18. KORELO, Raciele Ivandra Guarda et al. Efeito de um programa cinesioterapêutico de grupo, aliado à escola de postura, na lombalgia crônica. Fisioterapia em Movimento, v. 26, n. 2, p. 389-394, 2013.

https://doi.org/10.1590/S0103-51502013000200016

19. LIMA, Karen Bárbara Eloy; De Oliveira Carvalho, José Júnior. Auriculoterapia no tratamento da lombalgia. Textura, v. 13, n. 21, p. 133-139, 2019. doi.org/10.22479/desenreg2019v13n21p133-139

20. REAL, Bhetina Morena Martins Brito et al. Fisioterapia aquática no tratamento de dor lombar crônica. Revista Interdisciplinar de Saúde e Educação, v. 1, n. 1, p. 76-89, 2020.

21. SILVA, Dalvania Alves da et al. Analgesic efficacy of the association of cryotherapy and transcutaneous electrical nerve stimulation. BrJP, v. 1, n. 3, p. 274-278, 2018.

22. DA SILVA, Clícia Alves et al. Efetividade da terapia por estimulação elétrica em pacientes com dor lombar: revisão sistemática. Revista Cientefico, v. 16, n. 33, p. 147-166, 2016. doi.org/10.1590/S1413-35552011000300002

23. PESSOA, Diego Rodrigues et al. Association of facial massage, dry needling, and laser therapy in Temporomandibular Disorder: case report. In: CoDAS. Sociedade Brasileira de Fonoaudiologia, 2018. doi.org/10.1590/2317$1782 / 20182017265$.

24. Nascimento LDES, Nascimento KFES, Pessoa DR, Nicolau RA. Effects of Therapy with Light Emitting Diode (LED) in the Calcaneal Tendon Lesions of Rats: A Literature Review. ScientificWorldJournal. 2019 Feb 3;2019:6043019. doi: 10.1155/2019/6043019.

25. Lopes Silva RSD, Pessoa DR, Mariano RR, Castro ABS, de Oliveira RA, Ferraresi C. Systematic Review of 
Photobiomodulation Therapy (PBMT) on the Experimental Calcaneal Tendon Injury in Rats. Photochem Photobiol. 2020 Sep;96(5):981-997 DOI: 10.1111/php.13262

26. da Silva Leal MV, Lima MO, Nicolau RA, de Carvallho TMT, Abreu JAC, Pessoa DR, Arisawa EALS. Effect of Modified Laser Transcutaneous Irradiation on Pain and Quality of Life in Patients with Diabetic Neuropathy. Photobiomodul Photomed Laser Surg. 2020 Mar;38(3):138-144. doi: 10.1089/photob.2019.4714.

27. DE SANTANA, Josimari Melo et al. Revised definition of pain after four decades. BrJP, v. 3, n. 3, p. 197-198, 2020. doi.org/10.5935/2595-0118.20200191

28. VAN TILBURG, Mark L. et al. Feasibility of a stratified blended physiotherapy intervention for patients with non-specific low back pain: a mixed methods study. Physiotherapy Theory and Practice, p. 1-13, 2020.

29. BASFORD, Jeffrey R.; Sheffield, Charles G.; Harmsen, William S. Laser therapy: a randomized, controlled trial of the effects of low-intensity Nd: YAG laser irradiation on musculoskeletal back pain. Archives of physical medicine and rehabilitation, v. 80, n. 6, p. 647-652, 1999. doi.org/10.1016/S0003-9993(99)90167-3

30. KHOLOOSY, Leyla et al. Evaluation of the Therapeutic Effect of Low Level Laser in Controlling Low Back Pain: A Randomized Controlled Trial. Journal of Lasers in Medical Sciences, v. 11, n. 2, p. 120, 2020. doi: 10.34172/ jlms.2020.21

31. COTLER, Howard B. et al. The use of low level laser therapy (LLLT) for musculoskeletal pain. MOJ orthopedics \& rheumatology, v. 2, n. 5, 2015. doi: 10.15406/mojor.2015.02.00068

32. VALLONE, Francesco et al. Effect of diode laser in the treatment of patients with nonspecific chronic low back pain: a randomized controlled trial. Photomedicine and Laser Surgery, v. 32, n. 9, p. 490-494, 2014. https://doi. $\operatorname{org} / 10.1089 /$ pho.2014.3715

33. VANIN, Adriane Aver et al. Photobiomodulation therapy for the improvement of muscular performance and reduction of muscular fatigue associated with exercise in healthy people: a systematic review and meta-analysis. Lasers in medical science, v. 33, n. 1, p. 181-214, 2018.

34. LEAL-JUNIOR, Ernesto Cesar Pinto; Lopes-Martins, Rodrigo Álvaro Brandão; BJORDAL, Jan Magnus. Clinical and scientific recommendations for the use of photobiomodulation therapy in exercise performance enhancement and post-exercise recovery: current evidence and future directions. Brazilian Journal of Physical Therapy, v. 23, n. 1, p. 71-75, 2019. doi: 10.1016/j.bjpt.2018.12.002.

35. GLAZOV, Gregory et al. Laser acupuncture for chronic non-specific low back pain: a controlled clinical trial. Acupuncture in Medicine, v. 27, n. 3, p. 94-100, 2009. doi: 10.1136/aim.2009.000521.

36. HUANG, ZeYu et al. The effectiveness of low-level laser therapy for nonspecific chronic low back pain: a systematic review and meta-analysis. Arthritis research \& therapy, v. 17, n. 1, p. 1-8, 2015. DOI: 10.1186/s13075-015-0882-0 
37. AY, Saime; DOĞAN, Şebnem Koldaş; EVCIK, Deniz. Is low-level laser therapy effective in acute or chronic low back pain? Clinical rheumatology, v. 29, n. 8, p. 905-910, 2010. doi: 10.1007/s10067-010-1460-0.

38. KOLDAŞ DOĞAN, Şebnem; AY, Saime; EVCIK, Deniz. The effects of two different low level laser therapies in the treatment of patients with chronic low back pain: a double-blinded randomized clinical trial. Journal of Back and Musculoskeletal Rehabilitation, v. 30, n. 2, p. 235-240, 2017. doi: 10.3233/BMR-160739.

39. HSIEH, Ru-Lan; LEE, Wen-Chung. Short-term therapeutic effects of 890-nanometer light therapy for chronic low back pain: a double-blind randomized placebo-controlled study. Lasers in medical science, v. 29, n. 2, p. 671-679, 2014. doi: 10.1007/s10103-013-1378-2. 